MATEC Web of Conferences 23,01060 (2015)

DOI: $10.1051 /$ matecconf/20152301060

(C) Owned by the authors, published by EDP Sciences, 2015

\title{
Numerical Simulation of Unsteady and Periodic Motion Heat and Mass Transfer of a Highly Concentrated Granular Medium in a Coaxial Bunker
}

\author{
Natalia Chinchikeeva ${ }^{1 \mathrm{a}}$ and Alexsandr Shvab ${ }^{2}$ \\ ${ }^{1}$ National Research Tomsk State University, Physics-Technical Faculty, 634050 Tomsk, Russia \\ ${ }^{2}$ National Research Tomsk State University, Physics-Technical Faculty, 634050 Tomsk, Russia
}

\begin{abstract}
This paper presents numerical simulation of unsteady and periodic motion heat and mass transfer of a highly concentrated granular medium in a coaxial bunker. Mathematical model with variables vortex-stream functions was developed. Heat and mass transfer in highly concentrated granular materials at different time steps was studied for $\operatorname{Re}=0.25$ and $\operatorname{Pr}=10$ with periodically variable density of the heat flow.
\end{abstract}

\section{Introduction}

Currently widely are used pneumatic circulating apparatuses for mixing, drying, dosing and conveying of granular materials at high concentrations of the mixture components. Therefore, the urgent task is to develop mathematical models that describe the fluid flow, heat and mass transfer in highly concentrated granular materials.

\section{Physical and Mathematical model}

This paper present the flow of the dense layer in a plane channel of width $H$, which represents a coaxial tube disposed on the inner surfaces of the ribs for to intensify the process of mixing the mass and maintain the desired temperature (Fig. 1).

a Corresponding author: chinchikeeva@gmail.com 


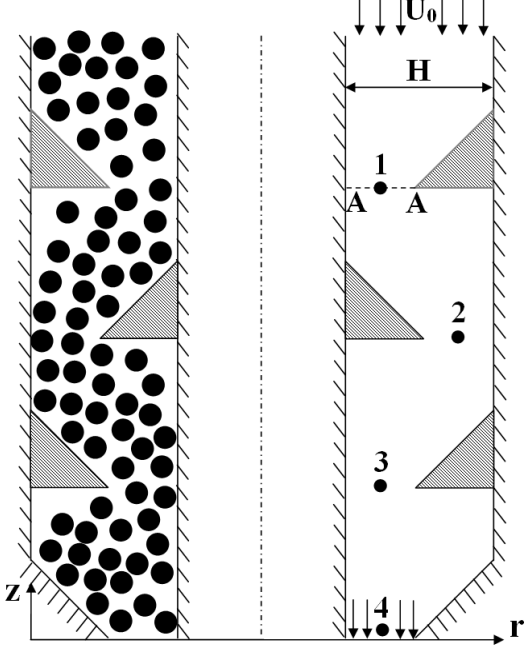

Figure 1. The study area.

The granular medium having a temperature $T_{0}$, comes from the top in a flat channel with a constant velocity $U_{0}$, and then the flow is heated by hot shelves; side walls which are have a periodically variable density of the heat flow by harmonic law

$$
q=q_{0}\left[1+A \sin \left(2 \pi t / t_{0}\right)\right] \text {. }
$$

Where $t_{0}$ is the period of oscillations of the heat flux at the wall, $t$ is the current time and $A$ - constant. Experimental and theoretical studies show that for describing the dynamics of the granular medium, we can use the laws of continuum mechanics [1-2]. In particular, to describe well-highly concentrated granular medium in a first approximation can be successfully applied by the theory of dynamics of viscous incompressible flow with the slip condition on the wall. As boundary conditions for a moving flow we use the condition of partial slip on the wall, which can be written by the form

$$
-\frac{\partial U_{s}}{\partial n}=\beta U_{s} ; \quad U_{n}=0 ;
$$

where $U_{s}, U_{n}$ are the tangential and normal components of the velocity vector in the direction to the wall, $n$ is normal, $\beta$ - slip coefficient of the medium on the wall, which varies from zero (full slip) to infinity (the condition of adhesion). Based on the above, the mathematical formulation of the problem in variables vortex - stream function can be represented as

$$
\begin{gathered}
\frac{\partial^{2} \psi}{\partial r^{2}}+\frac{\partial^{2} \psi}{\partial z^{2}}=\Omega r+\frac{1}{r} \frac{\partial \psi}{\partial r} \\
\frac{\partial \Omega}{\partial \tau}+\frac{\partial u_{r} \Omega}{\partial r}+\frac{\partial u_{z} \Omega}{\partial z}=\frac{1}{\operatorname{Re}}\left(\frac{\partial^{2} \Omega}{\partial r^{2}}+\frac{\partial^{2} \Omega}{\partial z^{2}}+\frac{1}{r} \frac{\partial \Omega}{\partial r}-\frac{\Omega}{r^{2}}\right) ; \\
\frac{\partial \Theta}{\partial \tau}+\frac{\partial u_{r} \Theta}{\partial r}+\frac{\partial u_{z} \Theta}{\partial z}=\frac{1}{\operatorname{Pr} \operatorname{Re}}\left(\frac{\partial^{2} \Theta}{\partial r^{2}}+\frac{\partial^{2} \Theta}{\partial z^{2}}+\frac{1}{r} \frac{\partial \Theta}{\partial r}\right)-\frac{\Theta u_{r}}{r} ;
\end{gathered}
$$

where, $\operatorname{Pr}=\nu / \alpha$ and $\operatorname{Re}=U_{0} H / \nu$ - Prandtl and Reynolds number, which are the matching parameters in the comparison of calculation results with experimental data [3].

To obtain a unique solution we use the boundary conditions presented in dimensionless form. At the inlet condition for $u_{y}=-1$, for stream function we have $\psi=x, \Omega=0$ and $\Theta=0$. At the outlet for defined functions we use the Neumann condition $(\partial / \partial y=0)$. At the left wall of the channel $\psi=0$ and at the right $\psi=$ const. The values of the vortex on the walls in accordance with equation (3) is determined by the 
decomposition of the flow function in a Taylor series near the wall taking into account the condition of slip (2). The result is

$$
\Omega_{w}=\frac{2\left(\psi_{w+1}-\psi_{w}\right)}{\Delta n^{2}}\left[\frac{\beta}{(\beta+2 / \Delta n)}\right] .
$$

Subject to the adhesion of the flow on the wall $(\beta \rightarrow 0)$ we have the condition of the Tom, and with the full slip of the medium at the wall $-\Omega w=0$. On the remaining walls of the streamlined body we use conditions full slip $(\beta \rightarrow)$. On the walls of the flat channel value of $\beta$ is determined from a comparison of numerical and experimental data. Here, the index w corresponds to the coordinates of the wall. For the temperature, we use the condition of the no heat flux $\partial \Theta / \partial \mathbf{n}=0$, with the exception of inclined shelves on which conditions are periodic changes of the density heat flow, which in dimensionless form is

$$
\frac{\partial \Theta}{\partial x}=-\left[1+A \sin \left(2 \pi \frac{t}{t_{0}}\right)\right]=-\left[1+A \sin \left(2 \pi \frac{\tau}{\mathrm{Ho}}\right)\right] ;
$$

where, Ho $=t_{0} U_{0} / H$ - the homochronicity criterion.

Numerical solution of the system (3)-(5) is carried out by the method for establishing of time using an implicit generalized scheme of alternating directions [4], and to the right-hand side of equation (3) is added the unsteady member. After finding stationary velocity field is solved the unsteady equation of heat transfer (5).

\section{Results}

Fig. 2 presents the temperature distribution depending on the time at four points 1-4 for periodic mode of movement of the granular medium. The graph is shown that the greatest temperature variations occur near the section A-A (Fig.1) and decrease downstream due to the thermal conductivity and convective heat transfer. On the same graph it is also possible to observe the initial phase establish the temperature over time in a given regions, are shown by dots. So for point 1 (Fig.1) exit on periodic mode is approximately two periods of oscillation for point 2 - four period, and for point 3 to six periods of the oscillations. The temperature at the outlet of the channel (curve 4) is set in eight periods, and fluctuations of temperature in this area are completely smooth. Fig. 3 and Fig. 4 presents the temperature distribution in the horizontal direction $\mathrm{x}$ in the section $\mathrm{A}-\mathrm{A}$ at different time steps. In Fig. 4 shows that the smallest temperature fluctuations occur near the plane wall and is increased in the direction to the ribs.

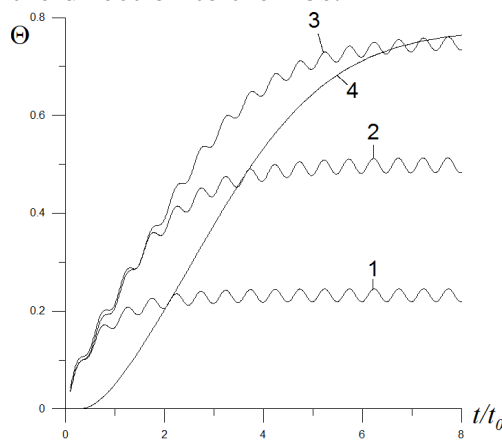

Figure 2. Temperature distribution of four points for follow parameters: $\mathrm{Re}=0.25 ; \quad \operatorname{Pr}=10 ; \quad \mathrm{Ho}=0.3 ; \quad A=0.5$; $\beta_{1}=120 ; \beta_{2}=0 ; \beta_{3}=0.5$

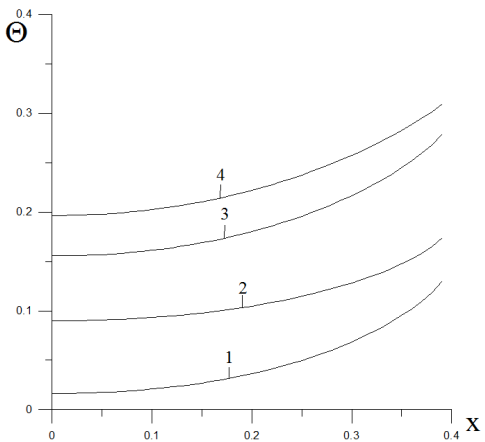

Figure 3. Temperature distribution in section A-A at diferent time steps for follow parameters: $\operatorname{Re}=0.25 ; \operatorname{Pr}=10$; $\mathrm{Ho}=0.3 ; \quad A=0.5 ; \quad \beta_{1}=120 ; \quad \beta_{2}=0$; $\beta_{3}=0.5$; Point $1-t / t_{0}=0.126$; Point $2-$ $t / t_{0}=0.504 ; \quad$ Point $3 \quad-\quad t / t_{0}=1.13$; Point $4-t / t_{0}=2.45$

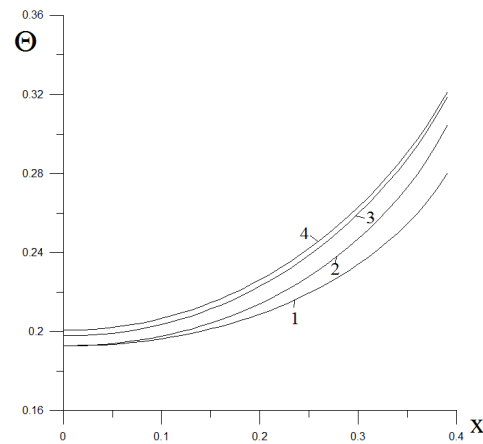

Figure 4. Temperature distribution in section A-A at diferent time steps for follow parameters: $\mathrm{Re}=0.25$; $\operatorname{Pr}=10 ; \quad \mathrm{Ho}=0.3 ; \quad A=0.5 ; \quad \beta_{1}=120 ;$ $\beta_{2}=0 ; \quad \beta_{3}=0.5 ;$ Point $1-t / t_{0}=3.02$; Point $2-t / t_{0}=3.09$; Point 3 $t / t_{0}=3.15$; Point $4-t / t_{0}=3.25$ 
The work is supported by RFBR (The Russian Foundation for Basic Research) grant 13-08-00372-a

\section{References}

1. The mechanics of granular media: Theory of fast motions. Collection of papers. Translation from English / I.V.Shirko. - M.: Mir (1985)

2. Shvab A.V., Martsenko M.S., Ryzhih Y.N. Modeling the hydrodynamics and the averaging process granular medium in the apparatus of powder technology // Engineer-nat. magazine. Vol.84, №4 (2011)

3. Z. R. Gorbis, Heat Transfer and Hydromechanics for Particle - Bearing Flows [in Russia], Moscow (1970)

4. Patankar C. Numerical methods for solving problems of heat transfer and fluid dynamics. M.: Energoatomizdat (1984) 